\title{
The Changes of Cognition in Teenagers after Playing Video Games
}

\author{
Kunsh K. Singh ${ }^{1}$ and Karen C. Molloy" ${ }^{1 \#}$ \\ ${ }^{1}$ Chantilly High School, Chantilly, VA, USA \\ "Advisor
}

$\underline{\text { ABSTRACT }}$

Recent studies have shown that video games alter cognition in teenagers, but it is unknown how teen cognition changes in the short-term directly after playing video games. This study measured the differences in selective attention, processing speed, sustained attention, and cognitive flexibility of video-game playing (VGP) adolescents at different time intervals after playing video games. Three different reasoning tests were used: the Paced Auditory Serial Addition Test (PASAT), the Sustained Attention to Response Task (SART), and the Stroop Task. A custom 250 question arithmetic test was also used, but was unable to uncover processing speed differences between VGPs after playing video games. All VGP participants in the study underwent reasoning tests before playing video games, played League of Legends (LoL) — known for its intensive use of spatial awareness, cognitive decision making, and working memoryfor an hour, and then took reasoning tests once again at different times after having played LoL. This experiment affirms that video games create cognitive enhancement, though the experiment uncovered that VGP teens have worsened selective and sustained attention as time elapses after playing video games. Future research may want to extend the variable of time to test how video games affect attention or cognition by time in a longer term with a larger sample size.

\section{Introduction}

With the development of the World Wide Web in 1989 by Sir Tim-Berners Lee, a surge of information became readily available across the globe. Eventually, video games, a form of entertainment where a user engages with a digital interface, were featured on the internet: known as internet games, these games increased the popularity of video games. Over 2 billion people worldwide play video games (Newzoo, 2017), and roughly 150 million individuals in the United States play video games (Entertainment Software Association, 2019). With a massive spike of interest in video games, many neuroscientists and psychologists wondered about the effects of video games on the human brain. They found that cognition, the use of the brain to think, perceive, or act, changes when people play video games. Video games involve the use of spatial cognition, where millions of sensory neurons in the eyes process visual information (Spence $\&$ Feng, 2010). However, the brain is incapable of interpreting all visual data and must enhance cognitive operations to detect specific images to remain spatially aware - by improving rapid image detection and spatial awareness, cognition is altered. (Spence \& Feng, 2010).

\section{Literature Review}

Over the past decades, research has been conducted on video games in conjunction with psychology and neuroscience. With the development of Magnetic Resonance Imaging (MRI), functional Magnetic Resonance Imaging (fMRI), and cognitive reasoning tests, researchers have been able to measure the impact of video games on the human psyche. 
Though some researchers claim that video games may be a segue toward mental disorder (King et al., 2017), others claim that video games may be a useful way of mental training (Green \& Bavelier, 2007).

Many previous studies have evaluated video games as a conduit towards cognitive disability. According to Simone Kühn, a well-published neuroscience expert of the Max Planck Institute for Human Development, the human reward system is altered with time spent playing video games (Kühn et al., 2011). In an experiment where video game playing (VGP) and non-video game playing (NVGP) individuals bet on randomized odds, he observed that a routine increase in time playing video games was positively correlated with lessened time spent consciously thinking about placing bets and left ventral striatum activity, a segment of the brain responsible for decision making and reward behavior (Kühn et al., 2011). An experiment done by other social science and neuroscience experts supports the strong correlation between video games and the reward system, and it also finds that wins generate a stronger response in the striatal, indicating changes in decision making. (Kätsyri et al., 2013). Additionally, psychiatric and brain science experts observed that gamers shown clips of video games demonstrated increased neural activity in their dorsolateral prefrontal cortex (DLPFC), right medial frontal gyrus (OFC), parahippocampal gyrus, and thalamus: such neural circuitry is associated with craving patterns, anticipation of a reward, and trouble distancing from stimulating activity, which is similar to the brain patterns of alcohol and nicotine abusers (Han et al., 2011).

With changes to the reward system, playing video games may lead to addiction. The term video game addiction (VGA), stemming from internet addiction disorder which causes certain traits like craving, euphoria, and tolerance (Dong et al., 2012), is deemed a mental disorder correlated with shyness and social detachment (Ayas, 2012). In a study conducted by clinical psychologists from Victoria University in Melbourne, Australia, VGA was found to cause stress, fearfulness, hopelessness, anxiety, and loss of self-esteem (Loton et al., 2016). This addiction has physical effects as well. VGA is responsible for stimulating neurotransmitters such as oxytocin, melanocortin, neurotensin, orexin, and Substance P (neurokinin A) (Tereshchenko et al., 2019). Aside from changes to the physical brain and cognition, video games affect gamers emotionally as well. Questionnaires from 2,891 VGP young adults reveal that many gamers act out of emotional avoidance and lack emotional coping strategies (Von der Heiden et al., 2019). Another 630 VGP adults claimed that playing video games enabled them to satisfy the craving of fulfilling something deep within themselves, losing track of real-world time (King et al., 2017).

Video games can also have other unwanted effects. Healthy German subjects introduced to video games had increased heart rates, reduced self-reported relaxation levels, and lower supplementary motor area (SMA) activity (Liu et al., 2019). However, reduced SMA activity could be explained by the VGP brain focusing its attention on a tighter scope of thought, similar to a video game training experiment done on NVGP people who became VGP individuals by renowned cognitive neuroscientist Daphne Bavelier, where decreased brain activity in the frontal-parietal network, which includes the SMA, was an indicator of increased brain efficiency and response time (Bavelier et al., 2012). Additionally, the VGP individuals had decreased visual crowding - indicating changes to the reticular network (Ackerman, 1992) - improving their ability to process visual information (Bavelier et al., 2012). This observed phenomenon where decreased brain activity led to increased brain efficiency can also be asserted in a study done by Stanford psychologists, where VGP graduate students had decreased activity in their medial prefrontal cortex and posterior cingulate cortex (Cole et al., 2012). However, the students also had increased activity in their motor cortex, visual cortex, and anterior insula, indicating heightened motor skills and visual processes (Cole et al., 2012).

Green and Bavelier's experiment found that training the brain with video games yields cognitive improvements. Green and Bavelier had NVGP people play video games eight hours a week up to a total of thirty hours, finding that they improved spatial cognition and decreased their visual crowding range (Green \& Bavelier, 2007). To measure such cognitive reasoning improvements, many researchers have tested VGP persons based on reasoning tests. In an experiment done by expert cognitive scientists Brian Glass, Todd Maddox, and Bradley Love, video game training improved mental intelligence and cognitive flexibility in twenty-six females, where cognitive ability was measured based on reasoning tests such as the Attention Networking Test (ANT) and the Stroop task (Glass et al., 2013). One specific type of training known as WM-REAS was found by cognitive psychologists to improve working memory the processing, utilizing, and storing of short-term information (Grabner \& Stern, 2010) - in teenagers, though it led 
to divided attention as well (Baniqued et al., 2014). Additionally, video games may have potential to enhance the cognitive ability of the elderly. In a study done by neurologists of the University of California, San Francisco, it was determined that elderly who cognitively trained with a racing game had an increase in brain wave activity from 4 to 7 $\mathrm{Hz}$ in the prefrontal cortex (Anguera, 2013 et al.). Though the researchers were unable to determine effects of working memory or sustained attention, the increase in theta waves in the prefrontal cortex does indicate that these individuals that went through video game training had an increased capacity to learn (Roohi-Azizi et al., 2017).

Furthermore, video games offer other cognitive benefits; Bavelier and other neuroscience doctorates claim that perceptual skills, spatial intelligence, and cognitive skills are benefitted by video games (Bavelier, 2011). Research done by neuroscientists and psychologists tested 128 individuals who played puzzle and speed-switching video games, using letter sets, form boards, paper folding, and matrix reasoning: the participants were found to have improved working memory and fluid intelligence after playing such video games (Kranz et al., 2017). Another experiment conducted by neurobiologists from the University of Irvine with 15 VGP people and 29 NVGP people found that 3D video games improved hippocampal plasticity and basic memory by testing individuals based on the Mnemonic Similarity Task (MST), virtual Water Maze task (vWM), and enumeration task (Clemenson et al., 2015). However, these cognitive enhancements may take a toll on other parts of the brain. Stanford psychologists utilized fMRI scans on 57 graduate students who played serious video game Re-Mission, determining that video games lead to a decrease of activity in the medial prefrontal cortex (Cole et al., 2012). Other research has found decreases in the DLPFC from violent video games based on blood oxygenation level-dependent (BOLD) activity (Aliyari et al., 2020; Hummer et al., 2010)— which can accurately depict neural activity (Hall et al., 2016). Nevertheless, these phenomenons of brain activity mirror brain patterns of attention deficit hyperactivity disorder (ADHD). Diminished activity in the prefrontal cortex - the center of attention, concentration, and behavior regulation - is a common indicator of ADHD determined by previous research (Arnsten, 2009). As such, video games appear to induce ADHD-like symptoms in the everyday cognition of VGP people.

Video games have been found to have different effects based on genre, and many researchers have uncovered how different types of video games affect the human brain. Iranian neuroscientists and engineers found that sports video games decrease salivary cortisol level, reducing stress levels, and increase reaction time (Aliyari et al., 2015). Alongside a decrease in stress, an increase in $3 \mathrm{~Hz}$ and $15 \mathrm{~Hz}$ brain waves with a decrease with 6 and $10 \mathrm{~Hz}$ was observed (Aliyari et al., 2015), stipulating a decrease in consciousness and complex thought, though creating a state of restful healing (Roohi-Azizi et al., 2017). The Iranian neuroscientists later found decreased salivary cortisol levels in participants who played puzzle games, and increased salivary cortisol levels (and thus heightened stress levels) in participants who played horror and runner games (Aliyari et al., 2018). Furthermore, violent video games have negative effects, increasing fluctuations in heart rate and thus decreasing cardiac coherence (Hasan et al., 2013). Using fMRI scans, Iranian clinical neuroscientists found violent gamers had increased BOLD activity and decreased DLPFC activity, which indicates a lowered cognitive ability. By measuring vectored brain waves with an electroencephalogram (EEG), the clinical neuroscientists unveiled that violent video games induce alpha-wave activity in the occipital and parietal lobes as well as beta-wave activity in the frontal cortex (Aliyari et al., 2020): alpha-wave activity can be attributed to relaxation when awake, while beta-wave activity can be attributed to alertness and conscious thinking (Roohi-Azizi et al., 2017). However, Chinese psychologists refuted that violent video games significantly affect spontaneous brain activity; they measured the amplitude of spontaneous low-frequency fluctuation (ALFF) and scanned the brains of VGP people using MRIs, concluding that violent video do not significantly affect spontaneous brain activity (Pan et al., 2018). In another study on 59 young adults conducted by Canadian cognitive scientists, it was determined that action video gamers had increased striatal gray matter and P3 components, resulting in greater sustained attention (West et al., 2015).

Most behavioral and cognitive studies regarding video games focus on young adults. However, few studies attempt to measure such effects on adolescents. In a study done by teenagers averaging an age of $18.86 \pm 1.46$ years old, it was found that gamers had improved processing speed, deductive reasoning, mathematical intelligence, and analogy scores based on the Wonderlic Cognitive Ability Test Questionnaire (Hisam et al., 2018). A different study 
done by health specialists including a psychiatrist and pediatricians tested VGP and NVGP adolescents of $12.5 \pm 1.9$ years old cognitively based on the Stroop Task, Trail Making Test, Part B (TMT-B), and California Verbal Learning Test for Children's Version (CVLT-C). They found that VGP adolescents had higher speeds when completing the reasoning tests, though they were faced with greater inaccuracies (Özçetin et al., 2019).

Also, scarce research attempts to quantify reasoning test scores based upon time. Diankun Gong, a clinical brain scientist part of the MOE Key Lab for Neuroinformation, found that increased time spent playing video games led to increased neural plasticity based on ALFF assessment based on BOLD signals and reasoning test assessment with the Raven Matrices Test (RMT) and IQ Testing (Gong et al., 2019). With a lack of research pertaining to how elapsed time after playing video games produces cognitive effects in adolescents, this raises the question: how does the cognitive ability of teenagers vary in the short-term after playing video games?

This research study adds to current literature, investigating how time elapsed after playing video games affects the cognition of adolescents by measuring reasoning test scores. As Özçetin states, "future studies could investigate more ranges of higher-order cognition, such as reasoning and everyday cognition" (Özçetin et al., 2019), whereas this study attempts to detect the shift in cognition between everyday cognition and cognition while playing video games in VGP adolescents. Unlike the study done by the health specialists, this study does not factor in time spent playing video games; the variable this study varies is the rest time between the end of playing video games and taking reasoning tests a second time, finding how the cognitive ability of VGP teens changes after playing video games. Boundaries presented by video games have been found to spur transient neural activity or short bursts of rapid neural activity (Cole et al., 2012), suggesting that video games will sustain effect on short-term cognition even after the player has quit. Since previous research indicates that video game players have increased sustained attention, neural plasticity, and processing speed when compared to non-video game players, it is important to see the duration of cognitive stimulation, and whether video games induce cognitive improvements in the short-term or long-term.

\section{Methods}

Eighteen VGP students $(n=18)$ and six NVGP students $(n=6)$ of total mean age 17.21 years were randomly selected from a sample of 50 high-school students to generalize how video games affect cognition in high-school adolescents. Students were recruited virtually, and all tests were held through Zoom with direct supervision from the researcher to prevent safety concerns from COVID-19. The cognitive ability of the NVGP students was measured with cognitive reasoning tests such as the PASAT, Stroop task, and SART taken in one sitting; VGP students were also instructed to take such cognitive tests as well as custom addition tests following a different procedure. To maintain consistency, all participants took reasoning tests in a specific order; the participants took the Stroop task, SART, PASAT, and then the custom addition test when applicable.

Prior to the experiment, all teens were to fill out an informed consent form, requiring their own consent as well as parental consent, and a pre-screening form, reporting the frequency at which they played video games. Individuals who had strictly no video game time on a weekly basis were considered as NVGP, while those who played over eight hours per week were considered as VGP.

Thirty minutes before the experiment began, all VGP teens were required to abstain from playing video games. Teen VGPs were first instructed to take a series of three reasoning tests consisting of the PASAT, Stroop Task, and SART. A select few whose computer systems met certain software criteria were instructed to take the custom addition test. Afterwards, each teenager played a free-to-play video game League of Legends (LoL) for an hour ( \pm 15 minutes to account for variable game durations), which was pre-installed on each participant's system prior to the start of the experiment. League of Legends was used since it challenges the player to not only strategize through spatial awareness, but also utilize working memory and rapid cognitive decision-making. After playing video games, each VGP student was randomly assigned to one of three experimental groups, taking the same reasoning tests again after resting zero minutes, five minutes, or fifteen minutes: this measure is the time between when VGP teens finish playing video games to when they take the reasoning tests a second time, referred to as elapsed time. While waiting to take 
such cognitive tests, participants were restricted from playing more video games or using electronic devices. Rather, each participant was engaged in group discussion with either other participants or the researcher about real-world topics, keeping their minds in a restful yet active state.

\section{Cognitive Reasoning Tests}

\section{Paced Auditory Serial Test (PASAT)}

The PASAT consists of 40 trials. Individuals will be shown two numbers and they must add those two numbers and produce an answer for the first trial. In all subsequent trials, participants will be presented with an additional number. They must remember the second of the previous two numbers and add that number to the newly presented number. If $\mathrm{n}_{0}$ Is the first number presented and $\mathrm{n}_{40}$ is the last number presented, we can represent the answers the subject should supply with the sequence $\mathrm{n}_{0}+\mathrm{n}_{1}, \mathrm{n}_{1}+\mathrm{n}_{2}, \mathrm{n}_{2}+\mathrm{n}_{3} \ldots \mathrm{n}_{39}+\mathrm{n}_{40}$.

The PASAT collects both accuracy and reaction speed; the number of correct answers, the 'time' being the average time taken to answer a question correctly, and the 'combined' is the average time it takes for the participant to answer any question whether it be correct or incorrect. As a result, the PASAT is indicative of processing speed, working memory, and cognitive flexibility.

\section{Stroop Task}

The Stroop Task also consists of 40 trials. For each trial, individuals will be presented a colored word. Each word will also be a name of a color. Individuals must identify the color of the word rather than the color that the word describes. For example, "red" should be identified as blue and "green" should be identified as red. The Stroop Task's intended goal is to measure processing speed, cognitive flexibility, and selective attention, though working memory is involved as well.

The Stroop Task determines three cognitive variables: congruent, incongruent and Stroop effect. The congruent is the average time in milliseconds it takes for a person to determine the color of a presented word that has the same written color as it is written (ex. blue) in while the incongruent is the average time in milliseconds it takes for a person to determine the ink-color of a word that specifies a different color (ex. red). Finally, the Stroop effect is the difference of the incongruent and congruent times, measuring response delay: the time it takes for a person to begin thinking after perceiving a stimulus.

\section{The Sustained Attention to Response Task (SART)}

SART will have 225 trials. In each trial, participants will be presented with a number 1 through 9 that will be quickly covered by a circle with a cross. If the number is not 3, the participants are tasked with pressing the space bar on their keyboard. This is known as a "Go trial (GT)." If the number is 3, participants must refrain from pressing their keyboard momentarily. This is known as a "No Go trial (NGT)." The accuracy of both 25 NGTs and 200 GTs from participants' SARTs which will be used to measure working memory, selective attention, and especially sustained attention.

\section{Custom Addition Test}

The custom addition test has infinite trials for a fixed duration of three minutes. Each trial consists of one single-digit addition question, where participants must do their best to answer the arithmetic question with accuracy. However, unlike all the reasoning tests above, a time limit rather than a question limit is present. Therefore, even though this test measures working memory, its greatest emphasis of measure is sustained attention and processing speed. 
Furthermore, a time limit with such arithmetic questions will be used to determine the participant's ability to apply a combination of sustained attention and processing speed.

\section{Ethics Statement}

All participants voluntarily provided their informed consent as well as parent or guardian acknowledgement to participate in this experiment. All personal data was stored on an encrypted hard drive to ensure safety of data. This study was approved by a school-based Institutional Review Board.

\section{Results and Discussion}

To reduce confounding factors such as intelligence or physical features, a matched pair analysis was taken, matching VGP participants' cognitive data before and after playing video games. Additionally, the difference in cognition was taken after VGP teens finished an hour of playing video game LoL, having waited a variable elapsed time or resting period $(0,5$, or 15 minutes) when having finished playing said video game.

A one-way Analysis of Variance (ANOVA) test was taken of all $0 \mathrm{~min}, 5 \mathrm{~min}$, and $15 \mathrm{~min}$ VGP participants to verify whether a significant difference in cognition exists before and after playing video games affirming previous studies. Additionally, ANOVA was used among the final cognitive results of each time group to verify whether cognitive ability fluctuates after a session of playing video games. Linear regression modeled the general trend of cognitive elements both before and after playing video games as well as among the three time groups after playing video games to model cognition after playing video games as a function of time.

Below demonstrates the results of each group for the conducted tests.
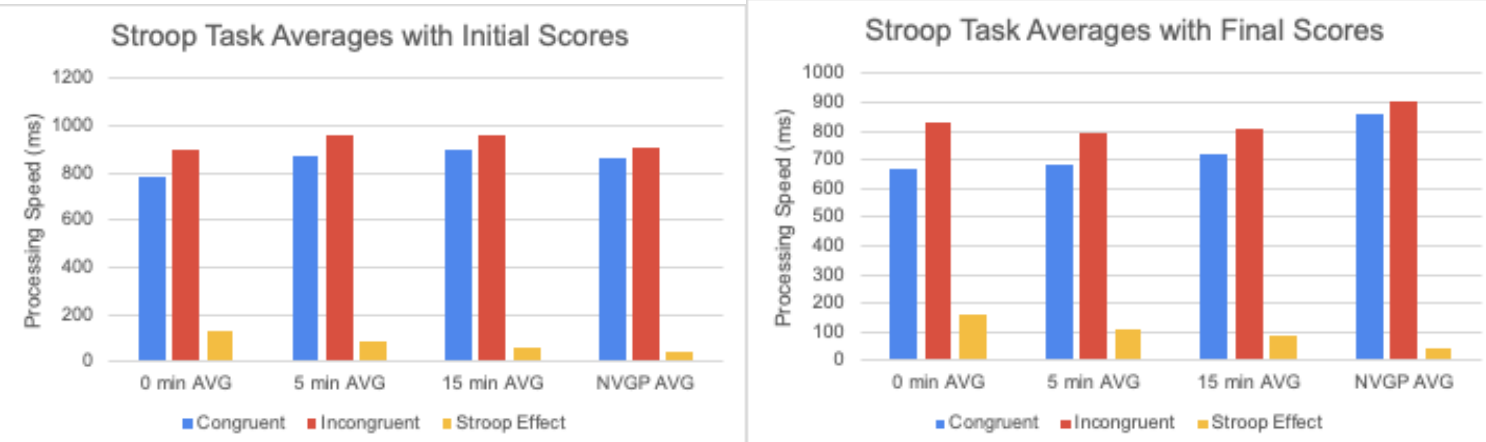

Figure 1.1. Stroop Task Results of NVGP participants versus VGP Participants

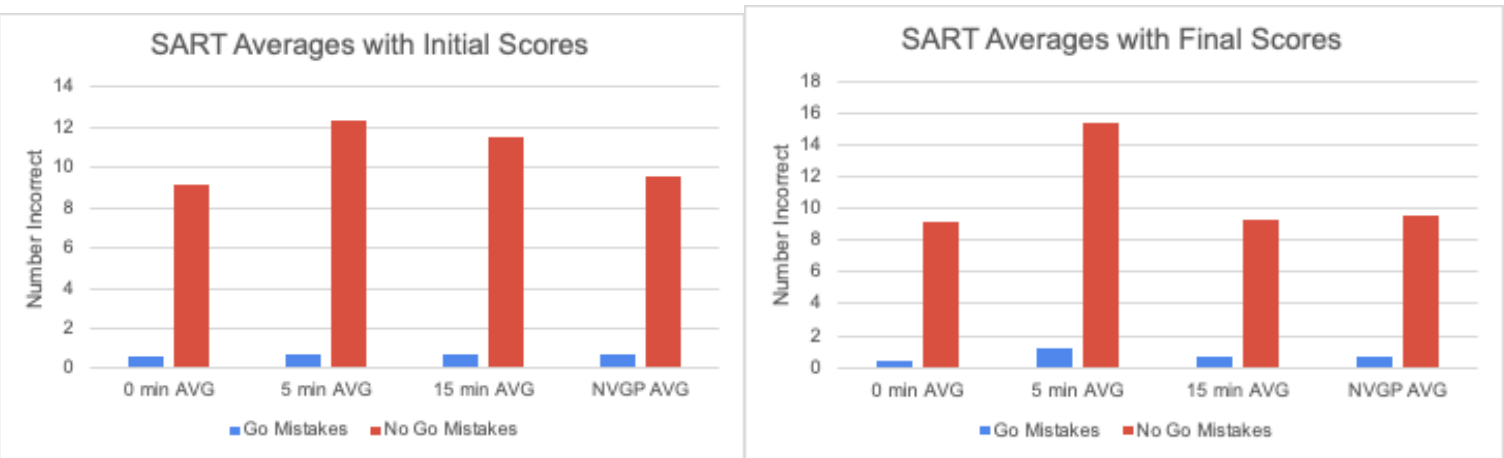

Figure 1.2. SART Results of NVGP participants versus VGP Participants 

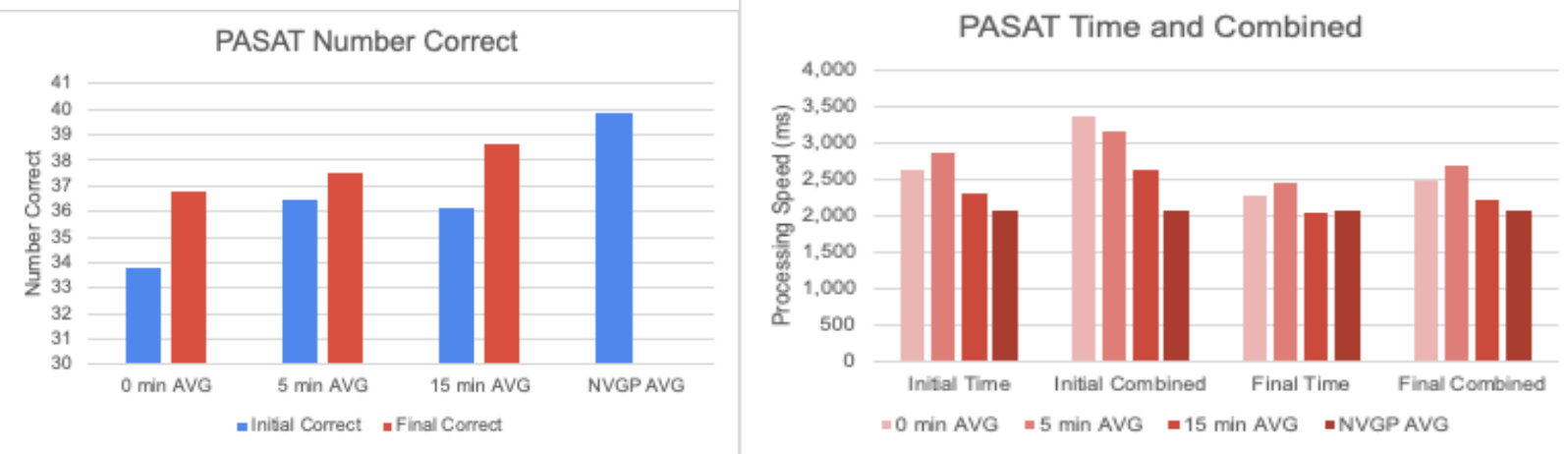

Figure 1.3. PASAT Results of NVGP participants versus VGP Participants

Figure 1.1, Figure 1.2, and Figure 1.3 compares the cognitive results of the NVGP participants to that of VGP participants in the Stroop task, SART, and PASAT tests respectively, measuring differences in everyday cognition. When comparing the NVGP results to VGPs some data points stick out. For example, when measuring everyday cognition using initial tests in VGPs by the PASAT (Figure 1.3), the average combined of the 0 minute group ( $x=3365$ ), 5 minute group ( $\mathrm{x}=3154)$, and the 15 minute group $(\mathrm{x}=2636)$ is substantially greater than that of the NVGP group $(\mathrm{x}=2347)$. With NVGP teens having a quicker combined time on average, NVGPs have better processing speed and cognitive flexibility when it comes to everyday cognition. However, after playing video games, VGP teens tend to outperform: the 0 minute group ( $x=2264)$ and the 15 minute group ( $x=2024)$ had better final combined times on average while the 5 minute group $(x=2447)$ did slightly worse than the NVGP teens. Similar results can be seen within the Stroop task (Figure 1.1), such that the initial VGP participant processing speed measures on average were slower than that of the NVGP participants, though the final VGP participant processing speed measures were faster than that of the NVGP participants.

The graphs in Figure 2 demonstrate the correlation between different processing speed measures (Congruent and Incongruent) in the Stroop before and after playing video games. Within Stroop task results after playing video games, a very flat correlation between time and both congruent and incongruent speeds can be observed with a high variance in the points (see Figure 1). When comparing groups to one another by standard deviation as per final congruent, the 0 minute group ( $\mathrm{SD}=186.7)$, the 5 minute group $(\mathrm{SD}=112.1)$ and the 15 minute group $(\mathrm{SD}=103.8)$ had tighter intervals over time, which supports the conclusion that processing speed in VGP teens is more variable when playing video games than when not. Similar results are present within the standard deviations in the final Stroop effect, such that the 0 minute group ( $\mathrm{SD}=65.7)$ and 5 minute group $(\mathrm{SD}=66.3)$ have significantly greater variance than the 15 minute group ( $\mathrm{SD}=37.6$ ), supporting that cognitive flexibility is more consistent as VGP teens transition into their everyday cognition. With tighter standard deviation, it can be implied that VGP teens were able to output a more consistent cognition with a clearer mind. Since working memory is directly influenced by mental clarity, the VGP teens therefore had improved working memory as time progressed after playing video games. Comparing the averages for Stroop effect after playing video games at 0 minutes $(x=160.8), 5$ minutes $(x=112.3)$, and 15 minutes $(x=87.8)$, a narrowing delay time in cognitive thinking is present within the VGP teens. A greater cognitive delay is indicative of utilizing higher levels of sustained attention, processing speed, and selective attention necessary - which VGP teens require to play video games - and thus it can be implied that VGP adolescents use less detailed thinking in their everyday life as opposed to when they play video games. 


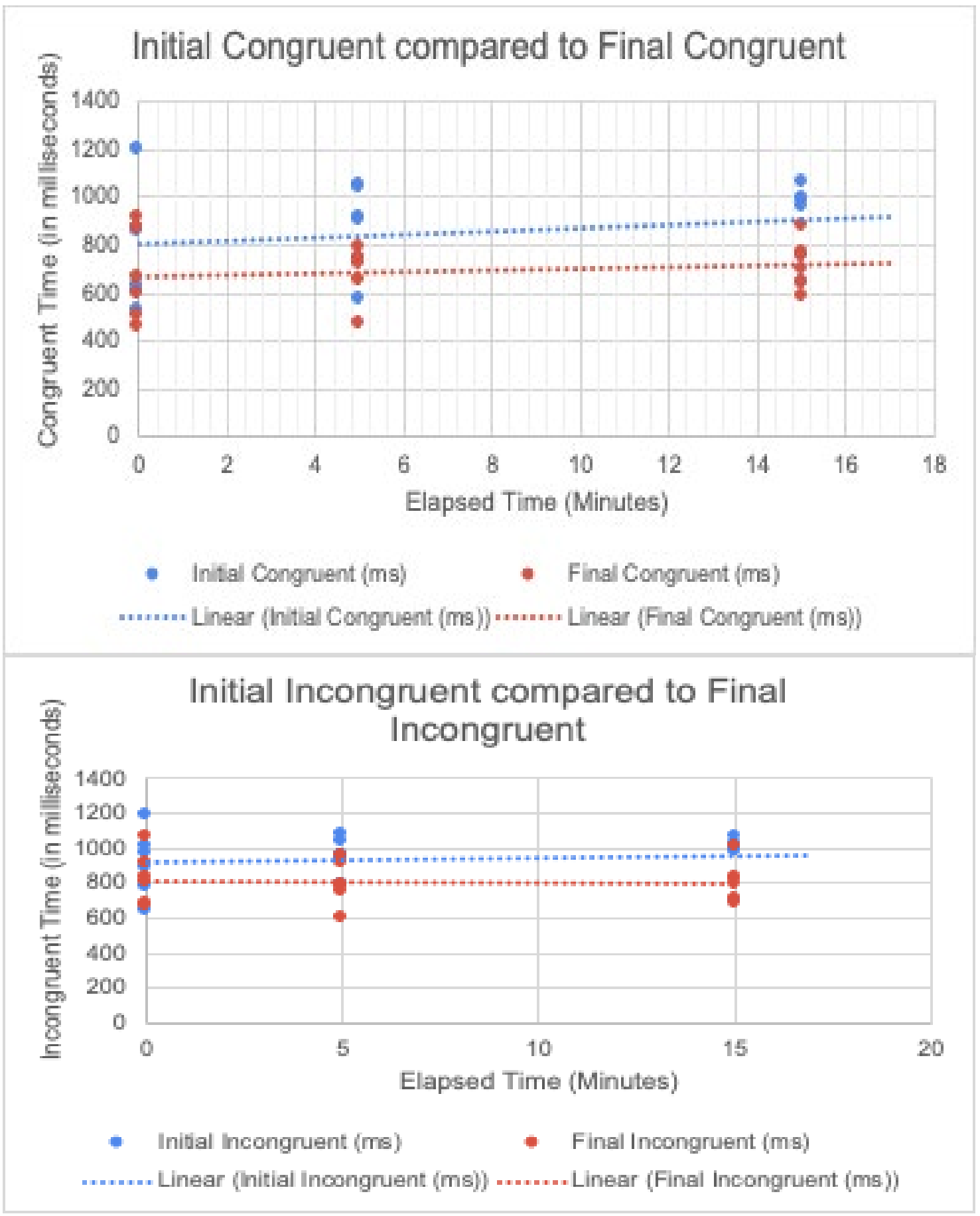

Figure 2. Stroop Task Results of VGP Teens Relative to Time After Playing Video Games 


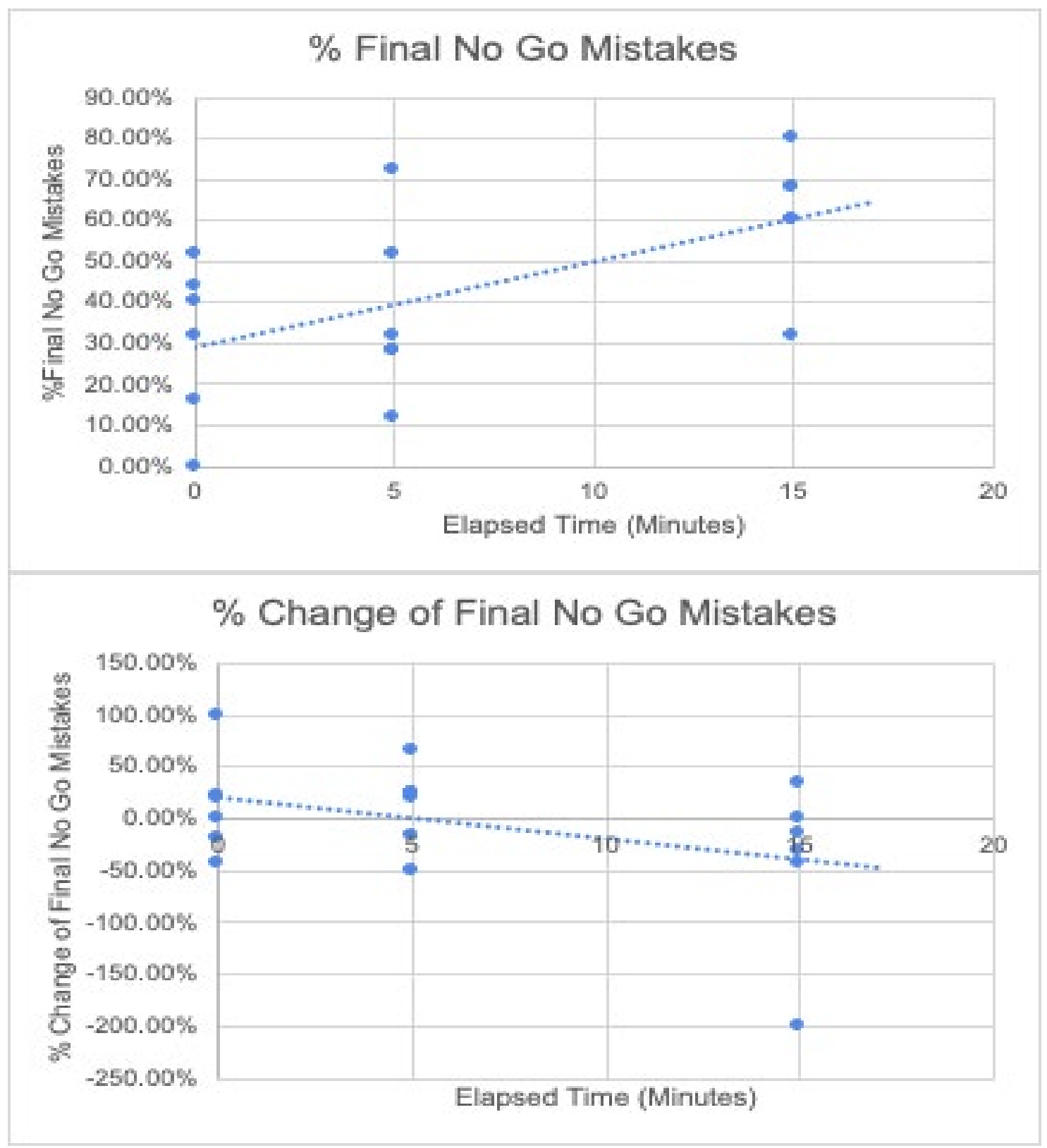

Figure 3. SART Results of VGP Teens Relative to Time After Playing Video Games

Figure 3 displays two graphs conveying the correlation between time and No Go Mistakes before and after playing video games. Though many participants disliked the length of the SART and some felt mentally fatigued after taking it, the SART precisely determined changes in VGP teens' cognition after playing video games by compelling the use of unconscious cognition. Compared to NVGP samples ( $\mathrm{x}=9.6 ; \mathrm{SD}=4.2)$, participants on average in the 0 minute VGP group ( $\mathrm{x}=9.2 ; \mathrm{SD}=4.8$ ) had less No Go Mistakes signaling that gamers at the time of playing video games have greater sustained and selective attentions. However, the 5 minute $(x=15.3 ; \mathrm{SD}=4)$ and 15 minute VGP group $(\mathrm{x}=9.3$; $\mathrm{SD}=5.3)$ had larger average NGT Mistakes than the NVGP group, such that NGT Mistakes in VGP teens increased as time elapsed with a moderate relationship $(\mathrm{p}<0.03 ; \mathrm{r}=0.59)$ - such correlation (see Figure 3 ) indicates that sustained and selective attention are greatest at the time of playing video games. Appending previous findings that determined improved attention in VGP teens immediately after playing video games (Cain et al., 2014), these results implicate that after having played video games, the sustained and selective attentions of VGP teens gradually 
deteriorate as time progresses (attention divides). Additionally, by such data, the sustained and selective attentions of NVGP teens are better than that of VGP teens not cognitively stimulated by video games.
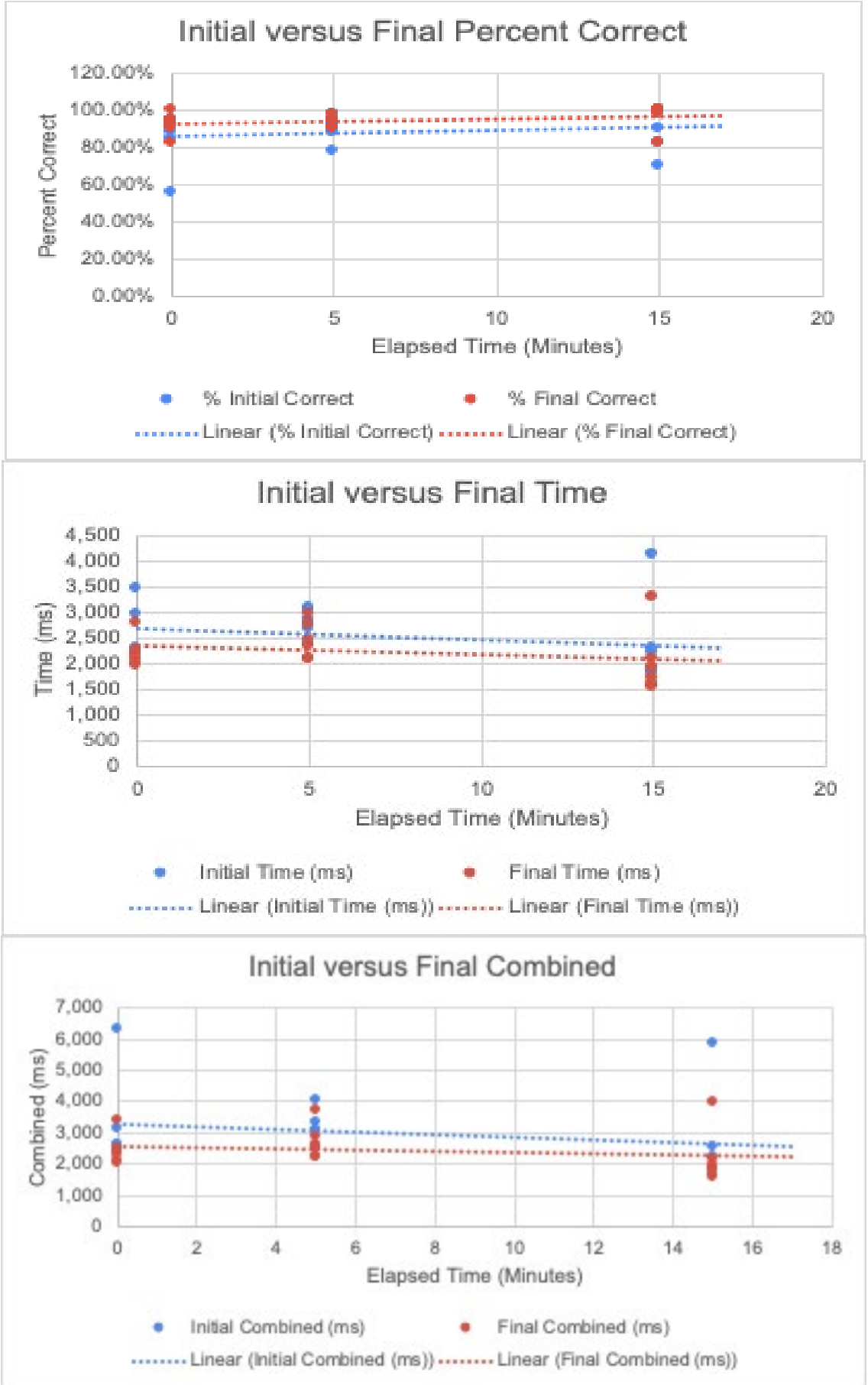

Figure 4. PASAT Results of VGP Teens Relative to Time After Playing Video Games

Figure 4 depicts three graphs that demonstrate changes in the percent correct, final time, and combined time respectively in VGP teens before and after video games. The percent correct $(\mathrm{p}<0.07 ; \mathrm{r}=0.69)$ increases after playing video games demonstrating an improved working memory and cognitive flexibility. Additionally, both the time 
$(\mathrm{p}<0.08 ; \mathrm{r}=0.88)$ and combined $(\mathrm{p}<0.1 ; \mathrm{r}=0.83)$ decreased slightly, indicating greater processing speed and working memory after playing video games.
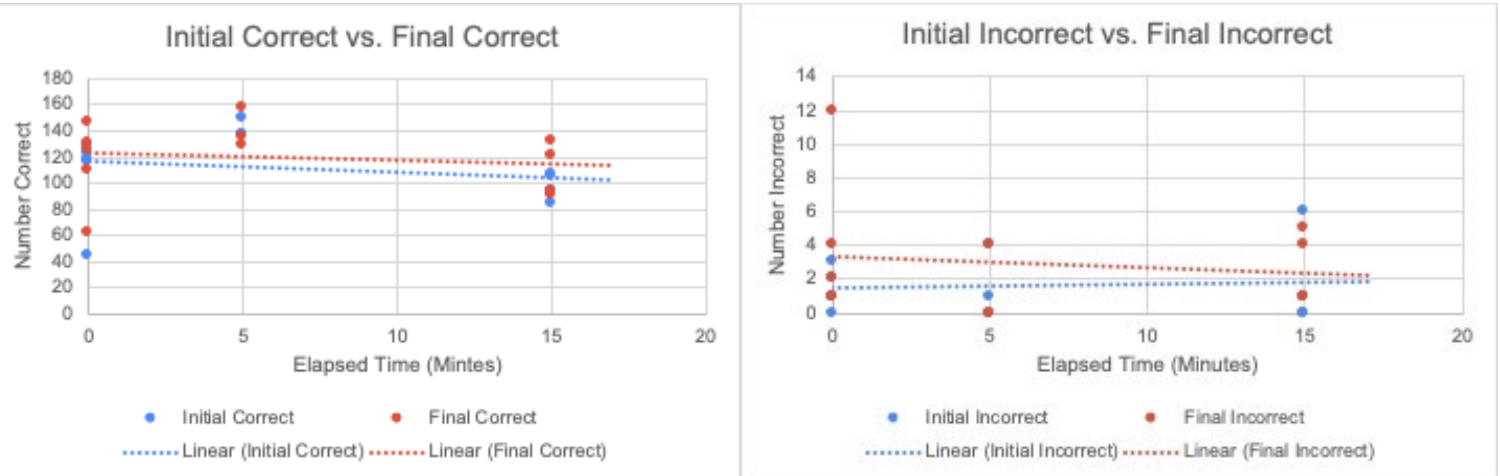

Figure 5. Custom Addition Test Results of VGP Teens Relative to Time After Playing Video Games

Figure 5 presents the findings from the custom addition test in a specified sample of $12 \mathrm{VGP}$ teens. However, minimal findings could be drawn since the change between initial and final correct $(\mathrm{p}>0.5 ; \mathrm{r}=0.91)$ and total correct $(\mathrm{p}>0.4 ; \mathrm{r}=0.89)$ lacked statistical significance.

\section{Conclusion}

This study examined changes in VGP teen cognition over time after playing video games. By assessing participants with reasoning tests before playing video games as well as after one of three resting intervals when finished playing video games, trends in cognition over time in VGP teens were formed. The reasoning tests that were used to assess cognition include the Stroop task, SART, and PASAT.

Analysis of the Stroop task's results (see Figure 2) before and after video games calls attention to improvements within the final congruent $(\mathrm{p}<0.01 ; \mathrm{r}=0.71)$ and incongruent $(\mathrm{p}<0.01 ; \mathrm{r}=0.73)$ values from their initial counterparts, asserting previous findings that video games improve short-term cognition (Pohl, 2014 et al.; Spence, 2010). Similarly, the results of the PASAT (see Figure 4) exhibit an increase in percent correct ( $<<0.07 ; \mathrm{r}=0.69$ ), and a decrease in time $(\mathrm{p}<0.08 ; \mathrm{r}=0.88)$ and combined $(\mathrm{p}<0.1 ; \mathrm{r}=0.83)$ within the VGP participants. With improvements in such Stroop task and PASAT scores, VGP teens were seen to improve their processing speeds, cognitive flexibilities, selective attentions, and working memory capabilities. Though VGP participants experienced a greater Stroop effect on average after playing video games, Stroop effect was not a statistically significant data field ( $\mathrm{p}>0.3)$ when considering VGP cognition before and after video games.

Though VGP teens may have higher levels of cognition when gaming, their sustained and selective attentions are significantly lower than NVGP teens as presented by the SART test scores when VGP adolescents transition to their everyday cognition. With an increase of divided attention in VGP teens (presumed by a decrease in selective attention and sustained attention) over time after playing video games seen by this experiment, as well as a drastic decrease in activity within the frontal-parietal network in VGP young adults observed by other studies (Bavelier et al., 2012), video games appear to instigate ADHD-like symptoms within VGP people. If such a phenomenon in young adults observed by Bavelier is mirrored in teens, then correlation between ADHD-like behavior (or possibly even ADHD itself) and VGP may exist. Yet, such divided attention may stem from lowered relaxation or increased heart rate found in another study (Liu et al., 2019), though such results are contradicted by Iranian neuroscientists who determined heightened alpha wave activity from video game players, signifying increased relaxation levels in VGP participants (Aliyari et al., 2020). Nonetheless, worsened selective attention and sustained attention induced by video games seem to have a prolonged effect as per the SART finding, which leaves this experiment open to further inquiry as to why the effect may occur, and what other implications that can be drawn from the resulting conclusion. Further 
research can determine the potential correlation between ADHD and video games through extensive cognitive testing and MRI scans on healthy VGP individuals without diagnosis before and after playing video games, such that a heavy emphasis on attention testing (both selective and sustained) is taken while referencing the DSM-5 for ADHD diagnosis.

\section{Limitations}

This experiment had large outliers that heavily influenced the statistical significance of certain data values. For instance, if the conducted Stroop task was rid 1 of its most extreme outliers in each data group (when comparing the percent change of cognitive test scores before and after playing video games among the three time groups), the congruent ( $\mathrm{p}>0.7$ ) would have $\mathrm{p}$-value $<0.3$, incongruent $(\mathrm{p}>0.9$ ) would have a $\mathrm{p}$-value $<0.15$, and Stroop effect $(\mathrm{p}<0.6)$ would have a p-value $<0.4$. Therefore, it is imperative for future research to contain a much larger population for this experiment to lessen the impact of heavy outliers in the data. On a related note, the Stroop and PASAT tests contained much fewer trials than the SART test and as a result yielded higher error when comparing the final cognition of the three time groups; future studies should use greater trials in these tests to ensure greater consistency in the data, reducing variance. In conjunction with a greater sample size, further levels of the independent variable time can be included in future research, such that the relationship between cognition in VGP teens and time can be mapped up to two hours after playing video games. By assessing a longer period, conclusions can be drawn to verify the accuracy of the relationship between divided attention and video games observed by this study and ascertain whether video games may be correlated with ADHD-like behavior.

\section{Future Research}

Other future research could determine the cognitive impacts video games have on neurotransmitters; further research could quantify dopamine, serotonin, vasopressin, and endorphin levels before and after video games to chart depths of craving or mood stabilization induced by playing video games. A different set of neurotransmitters including oxytocin, melanocortin, neurotensin, orexin, and Substance P (neurokinin A) are closely related with VGA (Tereshchenko et al., 2019): measuring such neurotransmitters on a quantitative basis may be indicative of the severity of VGA, and whether or not it operates on a physical level. Furthermore, another aspect worth considering in conjunction with neurotransmitters is time. By quantifying neurotransmitters relative to time, a clear impact will be seen on the way that video games stimulate the brain and whether that may be helpful or harmful to dopaminergic pathways both in the short term and long term. Additionally, quantifying neurotransmitters as per time will enable researchers to determine physical effects of video games; these findings may either assert the severity of VGA. or prove otherwise to be a potentially healthy way to stimulate the brain as a destressor and elate the mood of the player. Future research may be able to quantify neurotransmitter changes induced by video games using models of uncaging molecules (Shoham et al., 2005) or BOLD signals (Bruinsma et al., 2018).

\section{Acknowledgements}

I would like to thoroughly thank my teacher and advisor, Mrs. Karen C. Molloy, for her continuous motivation and support. 


\section{Bibliography}

Ackerman S. Discovering the Brain. (1992). Major Structures and Functions of the Brain. Washington (DC): National Academies Press (US). https://www.ncbi.nlm.nih.gov/books/NBK234157/

Aliyari, H., Sahraei, H., Daliri, M. R., Minaei-Bidgoli, B., Kazemi, M., Agaei, H., Sahraei, M., Hosseini, S., Hadipour, M. M., Mohammadi, M., \& Dehghani Mohammad Abadi, Z. (2018). The Beneficial or Harmful Effects of Computer Game Stress on Cognitive Functions of Players. Basic and Clinical Neuroscience, 9(3), 177-186. doi.org/10.29252/nirp.bcn.9.3.177

Aliyari, H., Kazemi, M., Tekieh, E., Salehi, M., Sahraei, H., Daliri, M. R., Agaei, H., Minaei-Bidgoli, B., Lashgari, R., Srahian, N., Hadipour, M. M., Salehi, M., \& Aghdam, A. R. (2015). The Effects of Fifa 2015 Computer Games on Changes in Cognitive, Hormonal and Brain Waves Functions of Young Men Volunteers. Basic \& Clinical Neuroscience, 6(3), 193-201.

Aliyari, H., Sahraei, H., Erfani, M., Mohammadi, M., Kazemi, M., Daliri, M. R., Minaei-Bidgoli, B., Agaei, H., Sahraei, M., Seyed Hosseini, S. M. A., Tekieh, E., Salehi, M., \& Farajdokht, F. (2020). Changes in Cognitive Functions Following Violent and Football Video Games in Young Male Volunteers by Studying Brain Waves. Basic \& Clinical Neuroscience, 11(3), 279-288. https://doi.org/10.32598/bcn.9.10.335

Anguera, J. A., Boccanfuso, J., Rintoul, J. L., Al-Hashimi, O., Faraji, F., Janowich, J., Kong, E., Larraburo, Y., Rolle, C., Johnston, E., \& Gazzaley, A. (2013). Video game training enhances cognitive control in older adults. Nature, 501(7465), 97-101. https://doi.org/10.1038/nature12486

Arnsten A. F. (2009). The Emerging Neurobiology of Attention Deficit Hyperactivity Disorder: The Key Role of the Prefrontal Association Cortex. The Journal of Pediatrics, 154(5), I-S43. https://doi.org/10.1016/j.jpeds.2009.01.018

Ayas, T. (2012). The Relationship between Internet and Computer Game Addiction Level \& Shyness among High School Students. Educational Sciences: Theory \& Practice, 12(2), 632-636.

Baniqued P. L., Kranz M. B., Voss M. W., Lee H., Cosman J. D., Severson J. \& Kramer A. F. (2014). Cognitive training with casual video games: points to consider. Front. Psychol. 4(1010). doi: 10.3389/fpsyg.2013.01010

Bavelier, D. et al. (2012). Neural bases of selective attention in action video game players. Vision Research, 61, 132-143. doi:10.1016/j.visres.2011.08.007

Bavelier, D., Green, C. S., Han, D. H., Renshaw, P. F., Merzenich, M. M., \& Gentile, D. A. (2011). Brains on video games. Nature Reviews. Neuroscience, 12(12), 763-768. https://doi.org/10.1038/nrn3135

Bruinsma, T. J., Sarma, V. V., Oh, Y., Jang, D. P., Chang, S. Y., Worrell, G. A., Lowe, V. J., Jo, H. J., \& Min, H. K. (2018). The Relationship Between Dopamine Neurotransmitter Dynamics and the Blood-Oxygen-LevelDependent (BOLD) Signal: A Review of Pharmacological Functional Magnetic Resonance Imaging. Frontiers in Neuroscience, 12, 238-245. https://doi.org/10.3389/fnins.2018.00238

Cain, M. S., Prinzmetal W., Shimamura A. P., \& Landau A. N. (2014). Improved control of exogenous attention in action video game players. Front. Psychol. 5(69). doi:10.3389/fpsyg.2014.00069

Clemenson, G. D., \& Stark, C. E. (2015). Virtual Environmental Enrichment through Video Games Improves Hippocampal-Associated Memory. The Journal of Neuroscience : The Official Journal of the Society for Neuroscience, 35(49), 16116-16125. doi.org/10.1523/JNEUROSCI.2580-15.2015

Cole, S. W., Yoo, D. J., \& Knutson, B. (2012). Interactivity and reward-related neural activation during a serious videogame. PloS one, 7(3). doi.org/10.1371/journal.pone.0033909

Dong, G., Huang, J., \& Du, X. (2012). Alterations in regional homogeneity of resting-state brain activity in internet gaming addicts. Behavioral and Brain Functions : BBF, 8(41). doi.org/10.1186/1744-9081-8-41

Entertainment Software Association (2019). 2019 Essential Facts About the Computer and Video Game Industry. https://www.theesa.com/wp-content/uploads/2019/05/2019-

Essential-Facts-About-the-Computer-and-Video-Game-Industry.pdf 
Glass, B. D., Maddox, W. T., \& Love, B. C. (2013). Real-Time Strategy Game Training: Emergence of a Cognitive Flexibility Trait. PLoS ONE, 8(8), 1-7. doi.org/10.1371/journal.pone.0070350

Gong D., Yao Y., Gan X., Peng Y., Ma W. \& Yao D. (2019). A Reduction in Video Gaming Time Produced a Decrease in Brain Activity. Front. Hum. Neurosci. 13(134). doi.org/ 10.3389/fnhum.2019.00134

Grabner, R., \& Stern, E. (2010). Measuring Cognitive Ability. Building on Progress: Expanding the Research Infrastructure for the Social, Economic, and Behavioral Sciences, 1, 753-768.

Green, C. S., \& Bavelier, D. (2007). Action-video-game experience alters the spatial resolution of vision. Psychological science, 18(1), 88-94. doi.org/10.1111/j.1467-9280.2007.01853.x

Hall, C. N., Howarth, C., Kurth-Nelson, Z., \& Mishra, A. (2016). Interpreting BOLD: towards a dialogue between cognitive and cellular neuroscience. Philosophical Transactions of the Royal Society of London. Series B, Biological sciences, 371(1705), 20150348. doi.org/10.1098/rstb.2015.0348

Han, Doug Hyun et al. (2011). Brain activity and desire for Internet video game play. Comprehensive Psychiatry, 52(1), 88-95. doi:10.1016/j.comppsych.2010.04.004

Hasan, Y., Bègue, L., \& Bushman, B. J. (2013). Violent Video Games Stress People Out and Make Them More Aggressive. Aggressive Behavior, 39(1), 64-70. doi.org/10.1002/ab.214

Hisam, A., Mashhadi, S. F., Faheem, M., Sohail, M., Ikhlaq, B., \& Iqbal, I. (2018). Does playing video games effect cognitive abilities in Pakistani children?. Pakistan Journal of Medical Sciences, 34(6), 1507-1511. doi.org/10.12669/pjms.346.15532

Hummer, T., Wang, Y., Kronenberger, W., Mosier, K., Kalnin, A., Dunn, D., \& Mathews, V. (2010). Short-Term Violent Video Game Play by Adolescents Alters Prefrontal Activity During Cognitive Inhibition. Media Psychology, 13(2), 136-154. doi.org/10.1080/15213261003799854

Kätsyri J., Hari R., Ravaja N. \& Nummenmaa L. (2013). Just watching the game ain't enough: striatal fMRI reward responses to successes and failures in a video game during active and vicarious playing. Front. Hum. Neurosci. 7, 278. doi.org/10.3389/fnhum.2013.00278

King, Daniel L. et al. (2017). Tolerance in Internet gaming disorder: A need for increasing gaming time or something else?. Journal of Behavioral Addictions, 6(4) : 525-533. doi.org/10.1556/2006.6.2017.072

Kranz, M. B., Baniqued, P. L., Voss, M. W., Lee, H., \& Kramer, A. F. (2017). Examining the Roles of Reasoning and Working Memory in Predicting Casual Game Performance across Extended Gameplay. Frontiers in Psychology, 8, 203. doi.org/10.3389/fpsyg.2017.00203

Kühn, S et al. (2011). The neural basis of video gaming. Translational Psychiatry, 1(11), e53. doi.org/10.1038/tp.2011.53

Liu, Shuyan, et al. (2019). Short-Term Effects of Video Gaming on Brain Response during Working Memory Performance. PLoS ONE, 14(10), 1-13. EBSCOhost, doi.org/10.1371/journal.pone.0223666

Loton, D., Borkoles, E., Lubman, D., \& Polman, R. (2016). Video Game Addiction, Engagement and Symptoms of Stress, Depression and Anxiety: The Mediating Role of Coping. International Journal of Mental Health \& Addiction, 14(4), 565-578. doi.org/10.1007/s11469-015-9578-6

Newzoo (2017). 2017 Global Games Market Report: Trends, Insights, and Projections Toward 2020. http://progamedev.net/wpcontent/uploads/2017/06/Newzoo_Global_Games_Market_Report_2017_Light.pdf

Özçetin, M., Gümüştaş, F., Çağ, Y., Gökbay, İ. Z., \& Özmel, A. (2019). The relationships between video game experience and cognitive abilities in adolescents. Neuropsychiatric Disease and Treatment, 15, 1171-1180. doi.org/10.2147/NDT.S206271

Pan, W., Gao, X., Shi, S., Liu, F., \& Li, C. (2018). Spontaneous Brain Activity Did Not Show the Effect of Violent Video Games on Aggression: A Resting-State fMRI Study. Frontiers in Psychology, 8, 2219. doi.org/10.3389/fpsyg.2017.02219 
Pohl C., Kunde W., Ganz T., Conzelmann A., Pauli P. \& Kiesel A. (2014). Gaming to see: action video gaming is associated with enhanced processing of masked stimuli. Front. Psychol. 5(70). doi.org/10.3389/fpsyg.2014.00070

Roohi-Azizi, M., Azimi, L., Heysieattalab, S., \& Aamidfar, M. (2017). Changes of the brain's bioelectrical activity in cognition, consciousness, and some mental disorders. Medical Journal of the Islamic Republic of Iran, 31(1), 307-312. doi.org/10.14196/mjiri.31.53

Shoham, Shy, et al. (2005). Rapid Neurotransmitter Uncaging in Spatially Defined Patterns. Nature Methods, 2(11), 837-843. EBSCOhost, doi.org/10.1038/nmeth793

Spence I, Feng J. (2010). Video Games and Spatial Cognition. Review of General Psychology. 14(2), 92-104. doi.org/10.1037/a0019491

Tereshchenko S, Kasparov E. (2019). Neurobiological Risk Factors for the Development of Internet Addiction in Adolescents. Behav. Sci, 9(6), 62. doi.org/10.3390/bs9060062.

Von der Heiden, J. M., Braun, B., Müller, K. W., \& Egloff, B. (2019). The Association Between Video Gaming and Psychological Functioning. Frontiers in Psychology, 10, 1731. doi.org/10.3389/fpsyg.2019.01731

West, G. L., Drisdelle, B. L., Konishi, K., Jackson, J., Jolicoeur, P., \& Bohbot, V. D. (2015). Habitual action video game playing is associated with caudate nucleus-dependent navigational strategies. Proceedings. Biological Sciences, 282(1808), 20142952. doi.org/10.1098/rspb.2014.2952 